\title{
COVID-19: el reto de la prevención y vacunación efectiva
}

\author{
COVID-19: the challenge of prevention \\ and effective vaccination
}

\author{
Mónica Sánchez-Cárdenas, * Vanessa Lerma-Sánchez,* \\ Camilo Trimiño-Monroy,* David A Valdés-Lagunes* \\ * Área de Nefrología del Trasplante. Departamento de Nefrología y Metabolismo Mineral. \\ Instituto Nacional de Ciencias Médicas y Nutrición «Salvador Zubirán».
}

\begin{abstract}
RESUMEN
La llegada de la pandemia por SARS-CoV-2 ha venido a transformar cada uno de los aspectos de la dinámica mundial, debido a su gran impacto social, económico, y en la morbilidad y mortalidad de la población. Ante la falta de un tratamiento efectivo, el contar con medidas de prevención adecuadas resulta crucial. Por un lado, la higiene de manos con agua y jabón o con desinfectante de manos a base de alcohol es la medida preventiva más simple, de bajo costo y la más usada alrededor del mundo para evitar la transmisión de COVID-19. Por otro lado, el uso de mascarillas por trabajadores de la salud y no trabajadores de la salud puede reducir el riesgo de infección por virus respiratorios entre 80 y $47 \%$, respectivamente. En el contexto de una nueva epidemia o pandemia, el contar de manera universal con vacunación altamente eficaz y segura se vuelve una prioridad. Lamentablemente, suele llevar de 12 a 18 meses el contar con vacunación disponible y comercializable a gran escala, por lo que tomar en cuenta las enseñanzas de epidemias previas, se vuelve crucial. Actualmente, al menos 10 diferentes vacunas se encuentran ya bajo estudio en humanos, en espera de probar eficacia y seguridad.
\end{abstract}

Palabras clave: Prevención, vacunación, SARS-CoV-2, mascarilla, pandemia.

\section{INTRODUCCIÓN}

La llegada de la pandemia por SARS-CoV-2 ha venido a transformar cada uno de los aspectos de la dinámica mundial debido a su gran impacto social, económico,

\begin{abstract}
The arrival of the SARS-CoV-2 pandemic has transformed every aspect of the global dynamics due to its great social, economic, morbidity and mortality impact. In the absence of effective treatment, having adequate preventive measures is crucial. Hand hygiene with soap and water, or with alcoholbased hand sanitizer is the simplest, low-cost and most widely used preventive measure worldwide to prevent transmission of COVID-19. On the other hand, the use of masks by health workers and non-health workers can reduce the risk of respiratory virus infection by 80 and $47 \%$ respectively. In the context of a new epidemic or pandemic, having universal access to highly effective and safe vaccination is a priority. Unfortunately, it usually takes 12 to 18 months to have vaccination available and marketable on a large scale, so taking into account learning from previous epidemics is crucial. Currently at least 10 different vaccines are already under study in humans, waiting to prove efficacy and safety.
\end{abstract}

Keywords: Prevention, vaccination, SARS-CoV-2, mask, pandemic.

y en la morbilidad y mortalidad de la población. Ante la falta de un tratamiento efectivo, el contar con medidas de prevención adecuadas resulta crucial. Revisaremos, a continuación, el impacto potencial de cada una de las medidas preventivas hasta ahora estudiadas, 
desde la forma más simple y eficaz al momento: el lavado de manos, hasta la forma más prometedora de prevención universal: la vacunación.

\section{HIGIENE DE MANOS}

La higiene de manos con agua y jabón o con desinfectante de manos a base de alcohol es la medida preventiva más simple, de bajo costo y la más usada alrededor del mundo para evitar la transmisión de COVID-19.1

La epidemia de COVID-19 ha venido a enfatizar la importancia de una óptima higiene de manos, pues ésta reduce la contaminación y el esparcimiento del virus SARS-CoV-2, que es el causante de la enfermedad. Dentro de los cuidados de la salud, una higiene de manos efectiva para prevenir infecciones asociadas e infecciones cruzadas y para reducir la propagación de microorganismos resistentes ha sido una de las prácticas que llevan muchos años llevándose a cabo. ${ }^{2}$ Por ejemplo, Nightingale, ya desde 1860, hacía un llamado a las enfermeras a tener una higiene de cara y manos frecuentes a lo largo del día. ${ }^{1}$

\section{Adherencia en el lavado de manos}

A pesar de ser una medida simple y de muy bajo costo, la adherencia del personal de salud a una correcta higiene de manos está muy por debajo de lo deseado. En lo que respecta a los cuidados de la salud, los llamados «deslices» o «lapsos» están vinculados con errores que se basan en habilidades, y es más frecuente que ocurran éstos cuando una tarea se vuelve muy familiar y va requiriendo cada vez menos conciencia en su ejecución. La manera de mejorar el apego es mediante la educación sobre cuáles son los momentos en que la higiene de manos debe realizarse, la disponibilidad de equipo en el punto de atención, como agua corriente, jabón, desinfectante de manos a base de alcohol, así como la facilidad para el personal de salud para tener un espacio donde guardar sus pertenencias. $^{1}$

¿Cuándo es necesaria la higiene de manos?

- Antes de tocar a un paciente.

- Antes de realizar procedimientos que requieran de asepsia.

- Después de exponerse a fluidos corporales (o haber estado en riesgo de exposición).

- Después de tocar a un paciente.

- Después de tocar los alrededores del paciente.
Además de esto, en situaciones de pandemia como la actual, se ha estipulado que la higiene de manos debe ocurrir después de removerse los guantes de látex, y que el usar guantes jamás debe reemplazar una adecuada higiene de manos. Los guantes deben reservarse para actividades específicas, como procedimientos invasivos, contacto con superficies estériles o piel no intacta y membranas mucosas, manipulación de dispositivos contaminados, y para actividades que se consideren de riesgo, como el exponerse a fluidos corporales. $^{3}$

\section{Higiene de manos en la práctica clínica}

Al estar en contacto con pacientes, el personal de salud debe asegurarse que su ropa no se extienda por debajo del pliegue del codo. Además, muchas guías de práctica locales sugieren que el uso de joyas debe limitarse a un anillo simple de matrimonio (evitarlo si es posible). Cualquier corte o solución de continuidad en la piel debe cubrirse con vendaje resistente al agua. Las uñas deben ir cortas y limpias, sin pinturas artificiales. Estas medidas han sido recomendadas, puesto que algunos estudios observacionales a pequeña escala demuestran que llevar anillos o uñas postizas se asocia con aumento en la portación de microorganismos y, en algunos casos, se ha asociado con el surgimiento de brotes por distintas cepas. ${ }^{3}$

El jabón debe obtenerse de un dispensador; no debe ser jabón en barra, ya que puede portar gérmenes, o en todo caso, puede usarse gel a base de alcohol. En caso de lavarse las manos con jabón, debe haber disponibilidad de hidratante y toallas desechables de papel.

¿Agua y jabón o gel a base de alcohol?

Ambos métodos, tanto el uso de agua con jabón como el gel a base de alcohol, son efectivos para la higiene de manos, aunque cada uno tiene sus propias limitaciones. Es importante que el personal de salud conozca las políticas locales de cada hospital para saber en qué momentos está recomendado cada uno de estos métodos.

Cuando las manos están visiblemente manchadas o potencialmente contaminadas con fluidos corporales y cuando se está dando atención a pacientes con vómito o diarrea, es preferible siempre realizar lavarse las manos con agua y jabón, independientemente de si se usaron guantes de látex. A lo largo de la pandemia de COVID-19, la información acerca del uso de alcohol en gel ha ido generándose con rapidez; las 
guías recomiendan una concentración de, por lo menos, $60 \%$ de etanol. ${ }^{4}$

\section{Técnica de higiene de manos con agua y jabón}

Cuando las manos se lavan con agua y jabón, no es sólo el jabón o el agua lo que elimina los microorganismos. También es importante que el personal de atención médica entienda que el proceso mecánico del lavado de manos ayuda a eliminar los contaminantes. Este efecto, combinado con el del agua tibia, promueve la apertura de los poros en la piel de las manos y la parte inferior de los brazos, ayudando a la eliminación de microorganismos. ${ }^{5}$

Antes de comenzar con la técnica, las manos y las muñecas deben estar completamente expuestas y deben estar libres de joyas y ropa de manga larga. ${ }^{3}$ El procedimiento para lavarse las manos con agua y jabón es el siguiente:

1. Abra los grifos del fregadero con los codos o los pedales si están provistos.

2. Verifique que el agua esté a una temperatura cálida y cómoda, y remoje las manos completamente desde la punta de los dedos hasta los antebrazos inferiores.

3. Dispense una o dos aplicaciones de jabón líquido en la palma de la mano.

4. Frote las manos para crear una espuma, esto debería tomar aproximadamente 10 segundos.

5. Siga las etapas de lavado de manos 2-7 de la guía de la OMS, tal como se describe en la Figura 1.6

6. Enjuague bien las manos usando sólo agua, asegúrese de que se elimine todo el jabón de las manos y los brazos.

7. Cierre los grifos con los codos o los pedales si se incluyen, o también puede usar una toalla de papel limpia para cerrar los grifos. No use las manos.

8. Seque las manos y los brazos con una toalla de papel limpia y deseche la toalla usada de acuerdo con la política local.

Si la piel de las manos está notablemente seca, se puede aplicar con moderación una crema hidratante para manos de un dispensador montado en la pared hasta que se absorba.

Todo el procedimiento debería tardar entre 40 y 60 segundos. Después de lavar las manos con agua y jabón, se deberán secarlas completamente. Esto es porque la transmisión de microorganismos es más probable cuando las manos están tibias y húmedas, lo que crea las condiciones ideales para el crecimiento microbiano. $^{7}$

Técnica de la higiene de manos con alcohol en gel

El gel para manos a base de alcohol funciona mediante la lisis y desnaturalización de las células de los microorganismos, reduciendo a niveles bajos la contaminación microbiana en las manos.

De igual manera que con el lavado con agua y jabón, antes de comenzar con la higiene de manos con gel a base de alcohol, las manos y las muñecas deben estar completamente expuestas y, por lo tanto, deben estar libres de joyas y ropa de manga larga. ${ }^{3}$ El procedimiento para la limpieza con gel para manos a base de alcohol es el siguiente:

1. Aplique una palma de gel a base de alcohol en las manos.

2. Frote las manos juntas, asegurándose de que el gel se extienda por todas las manos, dedos y brazos.

3. Siga las etapas de limpieza de manos 2-7 de la guía de la OMS, tal como se describe en la Figura $1 .^{6}$

4. Asegúrese de que las manos estén secas y de que no queden residuos de gel en las manos o los brazos inferiores. Si hay residuos, repita las etapas que se muestran en la Figura 1 hasta que las manos y los brazos estén secos.

No se debe requerir humectante después de usar gel de manos a base de alcohol, porque estas preparaciones a menudo contienen emolientes para prevenir la piel seca. Sin embargo, el personal de atención médica puede verificar los ingredientes del gel para verificar la presencia de uno. ${ }^{1}$

\section{USO DE MASCARILLAS A NIVEL COMUNITARIO}

Aquellas gotas respiratorias (de saliva o de moco, ya sea de la boca o de la nariz) implicadas en la transmisión de las infecciones son denominadas como gotas de flush o de Flügge, en honor al bacteriólogo e higienista alemán Carl Georg Friedrich Wilhem Flügge (1847-1923), quien demostró que, en minutos y en un discurso tranquilo, se rocían gotitas en el aire. Las gotas de Flügge son partículas diminutas expelidas al 
Duración de todo el procedimiento: 40-60 segundos

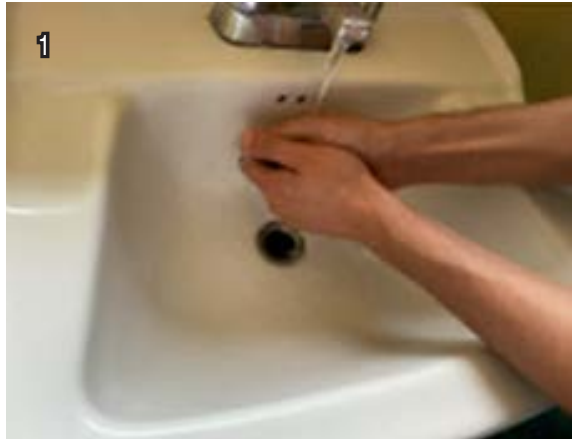

Mójese las manos con agua

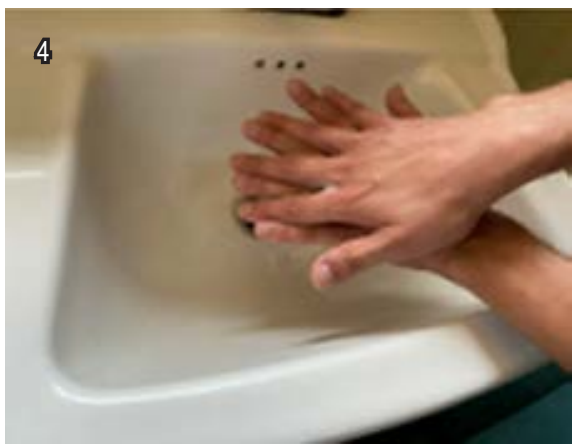

Frótese la palma de la mano derecha contra el dorso de la mano izquierda, entrelazando dedos y viceversa

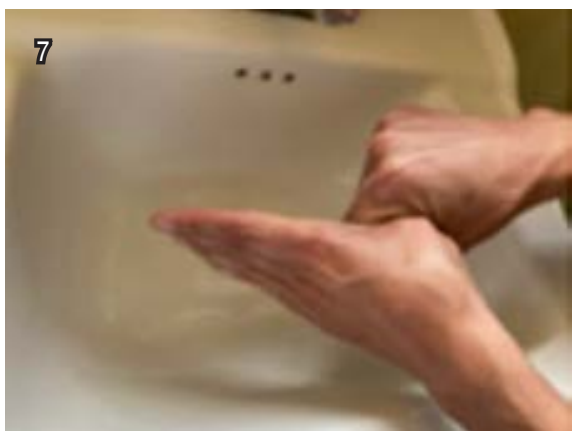

Frótese con un movimiento de rotación el pulgar izquierdo, atrapándolo con la palma de la mano derecha y viceversa

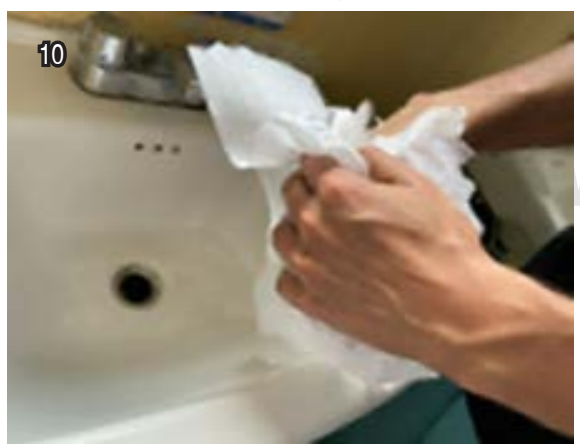

Séquese con una toalla desechable

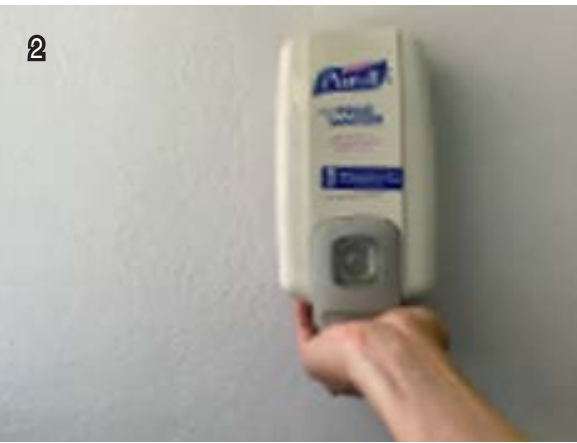

Deposite en la palma de la mano jabón suficiente para cubrir toda la superficie de las manos.

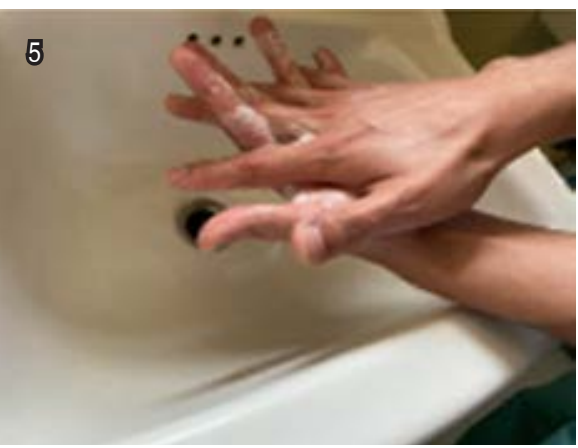

Frótese la palma de la mano entre sí, con los dedos entrelazados

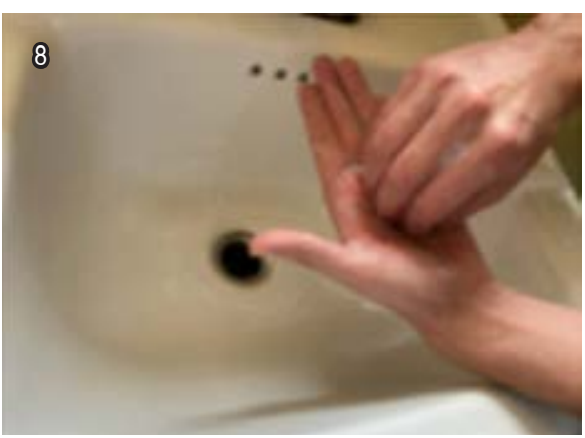

Frótese la punta de los dedos de la mano derecha contra la palma de la mano izquierda, haciendo un movimiento de rotación y viceversa

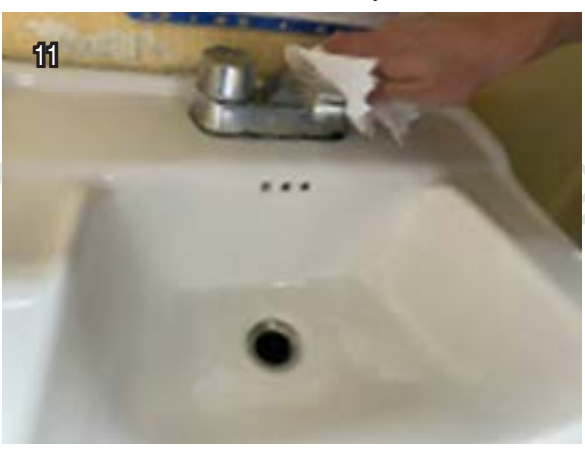

Sírvase de la toalla para cerrar el grifo

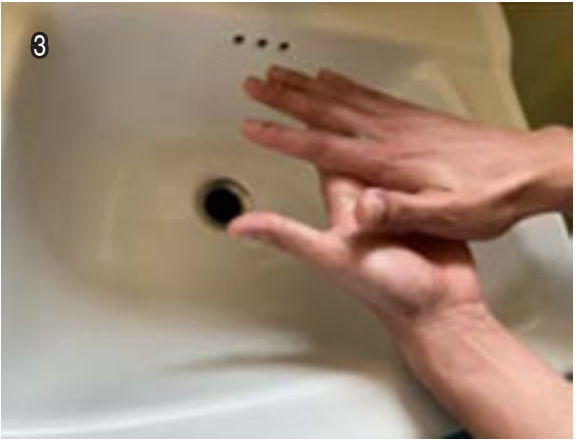

Frótese las palmas de las manos entre sí

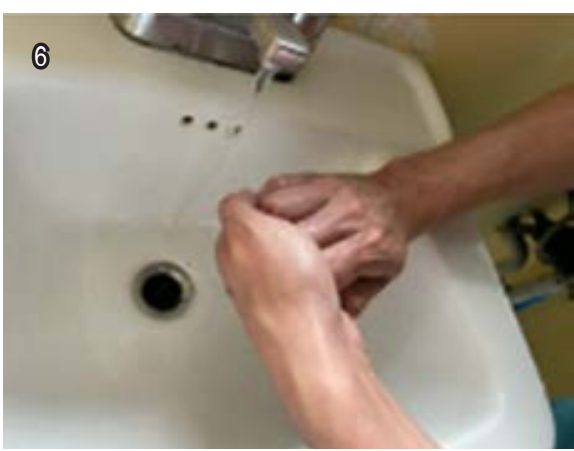

Frótese en dorso de los dedos de una mano con la palma de la mano opuesta, agarrándose los dedos

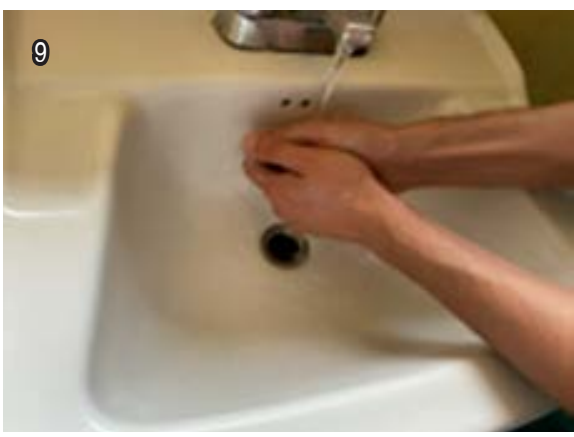

Enjuáguese las manos con agua

\section{2}

Figura 1:

Técnica de lavado de manos.

Adaptado de: World Health Organization (2009b) WHO Guidelines on Hand Hygiene in Health Care [último acceso el 5 de mayo 2020]. Disponible en: www.who.int/gpsc/5may/tools/9789241597906/en 
hablar, toser, estornudar y respirar, las cuales pueden traer gérmenes infecciosos de un individuo a otro. ${ }^{8}$

Las gotas pequeñas miden $10 \mu \mathrm{m}$ de diámetro, se producen durante el habla y al momento de toser; estas gotas contienen partículas virales, las cuales pueden mantenerse viables e infecciosas hasta por tres horas en el ambiente; éstas, asimismo, pueden transmitirse directamente a la vía aérea a través de aerosoles o indirectamente por contacto de lugares contaminados. La velocidad de movimiento de estas gotas es de 2-7 $\mathrm{m} /$ segundo al inicio de la tos y se tardan en caer en una superficie alrededor de nueve segundos. Las gotas grandes y visibles, por lo general, miden $500 \mu \mathrm{m}$ de diámetro, pero éstas no se mueven lejos por su peso, y rápidamente caen a las superficies por la fuerza de gravedad (en promedio 1 segundo). ${ }^{9}$

Las mascarillas faciales son un tipo de equipo de protección personal (EPP) que se usa para prevenir la diseminación de infecciones respiratorias. Estas mascarillas cubren la boca y nariz de quien las utiliza y, cuando se usan correctamente, pueden ser efectivas en ayudar en prevenir la transmisión de virus respiratorios y bacterias.

Existen dos tipos principales de mascarillas que se emplean para prevenir las infecciones respiratorias: mascarillas quirúrgicas (mascarillas faciales) y respiradores. Estas mascarillas se diferencian entre sí por el tipo y tamaño de partícula que son capaces de filtrar.

Las mascarillas quirúrgicas se usan más comúnmente para virus respiratorios que se propagan por gotas, las cuales viajan pequeñas distancias y son transmitidas por tos o estornudos. Éstas se adhieren suavemente a la cara, previenen que el usuario rocíe grandes gotas y que entren en contacto con la mano y la cara.

Los respiradores N95 bloquean 95\% de partículas aéreas. Éstos se adhieren de manera firme para prevenir la inhalación de partículas infecciosas pequeñas que se diseminan a través del aire en largas distancias después de que la persona infectada tose o estornude. Las enfermedades que requieren este tipo de respirador incluyen: tuberculosis, varicela, sarampión, entre otras. Éstos no se pueden sujetar con la barba o el bigote, o portarlas los niños, ya que no se obtiene la adherencia necesaria; más bien se vuelven candidatos para usar otro tipo de respiradores llamados «respiradores de aire asistido".

Si bien, tanto los respiradores N95 como las mascarillas faciales protegen de la transmisión de partículas virales, no hay diferencia entre usar mascarilla facial o respirador N95 en la comunidad (es decir, no trabajadores de la salud) para la prevención de in- fecciones causadas por virus, tales como influenza o SARS-CoV-2. Por ello, la Organización Mundial de la Salud (OMS) recomienda que personas que no sean trabajadoras de la salud, no usen respirador N95, ya que en pandemias suele haber desabasto de este material, el cual sí es requerido por trabajadores de la salud. ${ }^{10-12}$

Si se indica el uso de cualquier mascarilla o respirador, es importante el aseo de las manos como ya se mencionó. Después, se debe colocar la mascarilla sobre la nariz y la boca y se debe asegurar de que no haya espacios entre la máscara y su cara, verificando que esté bien sellada. Es muy importante evitar que se toque la mascarilla cuando se use. Si se llegase a tocar la mascarilla, es necesario lavarse las manos o utilizar un desinfectante (sanitización) para éstas. Cuando ya no se use la máscara facial o respirador, se debe retirar sin tocar la parte delantera de la misma y se debe desechar en un recipiente cerrado. Posteriormente, se debe lavar las manos nuevamente inmediatamente después de desechar la máscara facial.

Mclntyre y colaboradores demostraron que las personas que usaron mascarillas faciales tuvieron una reducción significativa del riesgo de infección clínica. A pesar de ello, piensan que es poco probable que el uso de las mascarillas en la comunidad sea una política de control eficaz para las enfermedades respiratorias estacionales.

Durante la epidemia de SARS (síndrome respiratorio agudo grave, por sus siglas en ingles) entre abril y mayo de 2003, en Hong Kong, la adherencia a los controles de infección fue alta: $76 \%$ de la población usó mascarilla facial, $65 \%$ lavó sus manos después de un contacto y $78 \%$ cubrió su boca cuando tosía o estornudaba. ${ }^{13}$

La adherencia al uso de mascarillas faciales pareciera tener un contexto cultural, por ejemplo, en países asiáticos se tiene mucha más adherencia al uso de mascarillas faciales. ${ }^{14}$ También se logra inferir que la adherencia al uso de la mascarilla depende de la comodidad de ésta. Jefferson y su equipo sugieren diseñar nuevas mascarillas faciales que mejoren su confort. ${ }^{15}$ Burgess y otros opinan que no sólo deben ser cómodas las máscaras, sino más fáciles de usar, pues mascarillas que sean fáciles de usar, cómodas y atractivas en apariencia, pueden hacer que las personas tengan más adherencia al uso de éstas. ${ }^{16}$

Se estima que el uso de las mascarillas faciales disminuye el riesgo relativo diario de adquirir una infección respiratoria en 60 a $80 \%$; cabe resaltar que esta reducción es dependiente del contexto, de la tem- 
porada y del virus en cuestión. El mecanismo exacto de la efectividad clínica del uso de mascarilla facial puede deberse a la prevención de inhalación de patógenos respiratorios, pero también tiene relación con el hecho de evitar el contacto mano-cara. Los resultados observados en estudios de cumplimiento y adherencia tienen relevancia global en la planeación del control de enfermedades respiratorias, especialmente enfocándose en el cuidado en el hogar. ${ }^{17}$ Cuando se presentan infecciones por nuevos virus emergentes, no hay opciones farmacéuticas disponibles, por lo tanto, las mascarillas juegan un rol importante para reducir la transmisión.

Liang y colaboradores, en su metaanálisis de 21 estudios, encontraron que el uso de mascarilla facial da un efecto protector ( $\mathrm{OR}=0.35$ y $95 \% \mathrm{IC}=0.24-0.51$ ). Ellos reportaron que el uso de mascarillas por trabajadores de la salud y no trabajadores de la salud puede reducir el riesgo de infección por virus respiratorios en $80 \%(\mathrm{OR}=0.20,95 \% \mathrm{IC}=0.11-0.37)$ y $47 \%(\mathrm{OR}=$ $0.53,95 \% \mathrm{IC}=0.36-0.79$ ), respectivamente; el efecto protector de usar mascarillas faciales en Asia (OR = $0.31)$ parece ser mayor que en países de Occidente $(\mathrm{OR}=0.45)$, y que las mascarillas tienen un efecto protector contra el virus de influenza $(O R=0.55)$, SARS $(\mathrm{OR}=0.26)$, y SARS-CoV-2 $(\mathrm{OR}=0.04) .{ }^{18}$

Las características físicas de las máscaras pueden prevenir el contagio del tracto respiratorio por virus. Leung y su equipo encontraron que las mascarillas quirúrgicas reducían significativamente la detección del ARN del virus de la influenza en gotas respiratorias y ARN de coronavirus en aerosoles. ${ }^{19}$ Liang y colaboradores demostraron que el uso de mascarillas por personas no infectadas puede prevenir efectivamente la diseminación de virus respiratorios, además de reducir el riesgo global de infecciones por virus respiratorios en $65 \% .{ }^{18}$ Este mismo beneficio se encontró no sólo en países asiáticos, sino en países occidentales.

Somsen y su equipo de investigación demostraron en su estudio que lugares con mejor ventilación reducen el tiempo en el aire de las gotas respiratorias. Por el contrario, lugares mal ventilados, por ejemplo, espacios poblados, como el transporte público u hogares geriátricos, son lugares de transmisión viral a pesar de la distancia social. La persistencia de pequeñas gotas respiratorias en estos espacios mal ventilados podría contribuir al esparcimiento de virus, especialmente al SARS-CoV-2. Este estudio sugiere que mejorando la ventilación de espacios públicos se diluye y se aclaran aerosoles potencialmente infecciosos. Por tanto, para disminuir la propagación del virus como SARS-CoV-2, se recomienda evitar espacios públicos mal ventilados tanto como sea posible. ${ }^{9}$

\section{EQUIPO DE PROTECCIÓN PERSONAL EN EL SECTOR SALUD}

El personal de salud requiere de precauciones adicionales para protegerse y prevenir la transmisión de SARS-CoV-2 en el entorno sanitario. Las precauciones que deben implementarse a los que atienden a este tipo de pacientes incluyen el uso de EPP de manera adecuada. Esto implica seleccionar el EPP correcto, como batas, guantes, cubrebocas, gafas y careta, así como también para aquellos procedimientos y respiradores, como el estándar N95 o FFP2, o equivalentes. Se debe educar a los profesionales de la salud sobre cuándo usar el EPP, así cómo los cuidados al ponerse, quitarse y cambiarlo para evitar la contaminación y cómo desechar y desinfectar adecuadamente este equipo.

En términos generales, el EPP es sólo una medida efectiva dentro de un ambiente con infraestructura adecuada, donde las instituciones de salud deben tener protocolos y políticas que describan el orden correcto sobre cómo ponerse y quitarse estos EPP de manera segura,$^{20}$ espacios adecuados que mantenga la distancia social entre pacientes y trabajadores de salud, salas bien ventiladas para pacientes con COVID-19 confirmados o sospechosos, entre otras. Estas medidas han sido descritas en la prevención de infecciones de la OMS y el control de infecciones respiratorias agudas propensas a epidemias y pandemias en la atención médica. Es importante mencionar que la ineficacia del EPP puede contribuir a la transmisión nosocomial de COVID-19. ${ }^{21}$

El orden para ponerse el EPP después de realizar la higiene de manos es: bata, máscara, gafas, careta y guantes. El orden para quitarse el EPP es: guantes, careta, gafas, bata y máscara, con aseo de manos entre cada paso. ${ }^{22}$ La OMS ha definido que el EPP debe usarse por el personal de salud según los procedimientos. ${ }^{23}$ Las recomendaciones sobre el uso de EPP se muestran en la Tabla $1 .{ }^{24}$

\section{Batas}

Se debe usar una bata de aislamiento limpia y ésta debe cambiarse cuando se contamine. Antes de abandonar estas áreas, el personal de salud debe quitarse la bata y desecharla en el contenedor de desechos rojos. Si utilizan batas reutilizables, éstas deben lavarse después de cada uso. ${ }^{25}$ 
Tipos de mascarillas faciales para el EPP

El término respirador o mascarilla de filtrado FFP2, FFP3 y N95 se utiliza para referirse a las máscaras de filtrado de alto rendimiento. La filtración se logra mediante una combinación de una red de microfibras de polipropileno y carga electrostática. Estas mascarillas deben ser resistentes a los líquidos cuando se usan con fines médicos.

Las máscaras FFP2, FFP3 y N95 no funcionan a menos que se ajusten bien a la cara y creen un sello; para ello, puede requerirse uso adicional de cinta adhesiva. La eficacia general del filtro de las máscaras FFP1, FFP2 y FFP3 es del 80, 94 y 99\%, respectivamente. ${ }^{26}$ Como tal, es probable que el FFP3 sea dos veces más efectivo que la máscara FFP2 y, en general, ambos son equivalentes o superiores a una máscara N95.

La mascarilla N95 significa que cumple con el estándar N95 del Instituto Nacional de Seguridad y Salud Ocupacional y del Centro de Control de Enfer- medades (CDC, por sus siglas en inglés) de Estados Unidos. Para avalar este criterio, la mascarilla debe bloquear, al menos, 95\% de las partículas de prueba de aerosol sólido y líquido. Las máscaras $\mathrm{N}, \mathrm{R}$ y $\mathrm{P}$ describen su resistencia creciente a los aceites, pero el número $(95,99$ o 100) se refiere al porcentaje mínimo de partículas filtradas en condiciones de prueba. ${ }^{27}$ La mascarilla N95 proporciona una protección contra partículas, pero no contra gases o vapores. En teoría, es un dispositivo diseñado para un solo uso y de duración de ocho horas continuas, pero debido a su demanda en algunos centros se ha generado un desabasto, por lo cual se han cuestionado y evaluado diversas estrategias para su reutilización. Existen cinco formas rápidas de matar un virus: irradiarlo, fumigarlo, calentarlo, vaporizarlo u hornearlo. Un boletín técnico de 3M (el mayor fabricante de N95 en Estados Unidos) estableció cuatro criterios que un buen método de descontaminación debe cumplir: 1) ser eficaz contra el organismo objetivo, 2) no dañar la filtración, 3) ser

Tabla 1: Tipo de equipo de protección personal (EPP) recomendado para prevenir el contagio de la enfermedad por SARS-CoVID-2.

\begin{tabular}{|c|c|c|c|}
\hline \multirow[t]{3}{*}{$\begin{array}{l}\text { Pacientes con } \\
\text { COVID-19 en cuartos } \\
\text { de hospitales }\end{array}$} & $\begin{array}{l}\text { Personal } \\
\text { de salud }\end{array}$ & Atención directa & $\begin{array}{l}\text { Batas } \\
\text { Máscara quirúrgica } \\
\text { Gafas/caretas guantes }\end{array}$ \\
\hline & & $\begin{array}{l}\text { Procedimientos de generación de aerosol } \\
\text { realizados en pacientes con COVID-19 }\end{array}$ & $\begin{array}{l}\text { Bata de manga larga } \\
\text { Máscara FFP2 } \\
\text { Gafas o caretas } \\
\text { Dobles guantes no estériles }\end{array}$ \\
\hline & $\begin{array}{l}\text { Trabajadoras } \\
\text { de limpieza }\end{array}$ & $\begin{array}{l}\text { Entrar a la habitación de pacientes con } \\
\text { COVID-19 }\end{array}$ & $\begin{array}{l}\text { Bata } \\
\text { Máscara FFP2 } \\
\text { Gafas o caretas } \\
\text { Guantes }\end{array}$ \\
\hline $\begin{array}{l}\text { Ambulancia o trans- } \\
\text { porte de pacientes con } \\
\text { COVID-19 }\end{array}$ & $\begin{array}{l}\text { Trabajadores } \\
\text { de salud }\end{array}$ & Transporte de pacientes con COVID-19 & $\begin{array}{l}\text { Bata de manga larga } \\
\text { Máscara FFP2 } \\
\text { Gafas o caretas } \\
\text { Dobles guantes no estériles }\end{array}$ \\
\hline $\begin{array}{l}\text { Instalaciones para } \\
\text { pacientes ambulatorios }\end{array}$ & $\begin{array}{l}\text { Trabajadores } \\
\text { de Salud }\end{array}$ & Pacientes con síntomas respiratorios & $\begin{array}{l}\text { Bata } \\
\text { Máscara FFP2 } \\
\text { Gafas o caretas } \\
\text { Guantes }\end{array}$ \\
\hline Sala de espera & Pacientes & 2 & $\begin{array}{l}\text { Pacientes con síntomas respiratorios deben usar cubrebocas. S } \\
\text { es posible aislarlos y mantener una distancia de } 1 \mathrm{~m} \text { cada uno }\end{array}$ \\
\hline Laboratorio & $\begin{array}{l}\text { Personal } \\
\text { de laboratorio }\end{array}$ & $\begin{array}{l}\text { Trabajando con muestras de secreciones } \\
\text { respiratorias }\end{array}$ & $\begin{array}{l}\text { Bata de manga larga } \\
\text { Máscara FFP2 } \\
\text { Gafas o caretas } \\
\text { Dobles guantes no estériles }\end{array}$ \\
\hline
\end{tabular}

Adaptado de: A alar C, Öztürk Engin D. Protective measures for COVID-19 for healthcare providers and laboratory personnel. Turk J Med Sci. 2020; 50 (SI-1): $578-584$. 
seguro para la persona que lo usa y 4) que no afecte el ajuste de la mascarilla. ${ }^{20}$ Sin embargo, a partir del 27 de marzo, 3M continuó insistiendo en que ningún método de desinfección ha cumplido con los cuatro criterios mencionados anteriormente cuando se repite de cinco a 10 veces. En una actualización de principios de abril, el fabricante afirma que, si bien el peróxido de hidrógeno y otros métodos de descontaminación aún se están evaluando, no recomienda hornear, vaporizar o esterilizar en autoclave debido a una degradación significativa del filtro. ${ }^{20}$

La base de evidencia para el uso de un tipo de máscara (por ejemplo, FFP3/FFP2/N95) sobre otra no es tan sólida como podría considerarse. Aún falta de evidencia contundente sobre el beneficio de las máscaras de alta filtración. ${ }^{26}$

\section{Protección de ojos}

La transmisión a través de los ojos no es segura para COVID-19, pero se ha demostrado en experimentos con animales que la transmisión de esta manera es posible. ${ }^{28}$ Por lo tanto, la protección ocular no debe descuidarse y debe considerarse como parte del EPP. ${ }^{23}$

\section{Guantes}

Una vez dentro de las habitaciones de los pacientes o áreas de atención, el personal de salud debe usar guantes limpios y no estériles. Al salir de la habitación del paciente o de las áreas de atención, se deben quitar los guantes y garantizar la higiene de las manos. ${ }^{25}$ Es importante señalar que los guantes no deben lavarse ni reutilizarse. ${ }^{29} \mathrm{Al}$ estar manipulando diferentes objetos que requiera el EPP, se debe enfocar a prevenir la infección cruzada.

\section{Prevención de infección cruzada}

Es importante recordar que el EPP es sólo una parte de un sistema para prevenir la contaminación de aquéllos que trabajan cerca de pacientes con COVID-19, lo que podría representar un riesgo tanto para este personal como para otro personal que allí labora y otros pacientes. Los elementos para reducir estos riesgos incluyen: lavado de manos de forma escrupulosa, lavado e higiene personal, regímenes de limpieza al menos dos veces al día para descontaminar superficies y equipos. Es muy importante que el personal de salud no toque los protectores oculares y las máscaras, entre otras cosas. Asimismo, es prioritario que el personal de salud conozca la variedad de factores que determinan el uso apropiado de los equipos de protección personal. Un adecuado manejo del EPP proporcionará al trabajador una posibilidad baja de contaminación con el virus.

\section{DESARROLLO DE VACUNAS PARA SARS-COV-2}

En el contexto de una nueva epidemia o pandemia, el contar de manera universal con vacunación altamente eficaz y segura, se vuelve una prioridad, por el impacto en el número de vidas perdidas y el deterioro económico vinculado con el retraso de su disponibilidad. ${ }^{30}$ Lamentablemente, suele llevar de 12 a 18 meses el contar con vacunación disponible y comercializable a gran escala, por lo que tomar en cuenta las enseñanzas de epidemias previas se vuelve crucial. ${ }^{31}$

\section{Aprendizaje de las experiencias previas}

Quizás la mayor parte de las expectativas relacionadas con la vacunación para SARS-CoV-2 derivan de la experiencia adquirida en la influenza. Así, la vacuna mayormente estudiada en receptores de trasplante sólido es, justamente, la vacunación contra influenza.

La respuesta a la vacunación suele medirse por medio de la inhibición a la hemaglutinación, en donde títulos de, al menos, 1:40 otorgan protección específica de $50 \%$ en el caso de población no inmunocomprometida (seroprotección). ${ }^{32} \mathrm{~A}$ la fecha, se desconoce el título específico que se considera como protector en el contexto de trasplantes; de esta manera, a pesar de que se ha demostrado la presencia de células $T$ específicas contra influenza, se incrementan postvacunación. Actualmente, se reconoce que la mayor parte de la protección adquirida deriva de la inmunidad humoral.

De esta manera, considerando el cambio en el título de anticuerpos (definiendo seroconversión como un incremento de cuatro veces en el título de anticuerpos), de 43 a $90 \%$ de los pacientes con trasplante, alcanzarían los valores protectores. ${ }^{33,34}$ Los pacientes que requieren más de $2 \mathrm{~g}$ al día de micofenolato de mofetilo, 0 mayores de 65 años, podrían presentar particularmente mala respuesta inmunológica tras la vacunación.

Dentro de las estrategias utilizadas, en afán de mejorar la respuesta inmunológica, se encuentran el uso de altas dosis de antígeno, uso de adyuvantes, aplicación intradérmica o el uso de dosis de refuerzo dentro 
de la misma temporada. De momento, ninguna de estas intervenciones se encuentra aprobada por la Food and Drug Administration (FDA) para su uso en receptores de trasplante dado lo limitado de la evidencia. ${ }^{32}$

\section{Búsqueda de la vacuna para SARS-CoV-2}

Al enfrentarnos con un patógeno viral con tan alta transmisibilidad y mortalidad asociada, el paso lógico siguiente es el desarrollo de una vacuna altamente efectiva, segura y que permita la vacunación a gran escala. Sin embargo, contar con tal herramienta resulta costoso, requiere tiempo y planeación sustancial para garantizar su seguridad.

Alrededor del mundo, hasta el momento, más de 90 vacunas distintas contra SARS-CoV-2 están en desarrollo por diferentes grupos de estudio. De ellos, al menos seis grupos de estudio han iniciado ya la aplicación de sus fórmulas en voluntarios sanos dentro de estudios de seguridad. ${ }^{35}$ Al día 3 de junio de 2020, se cuentan con 10 estudios clínicos con registro formal en el sitio clinicaltrials.gov y en reclutamiento activo (Tabla 2).

Dentro del tipo de vacunas desarrollados al momento, una proporción importante está representada por vacunas basadas en el uso de fragmentos de proteínas derivadas de coronavirus, o bien fragmentos de proteínas que imiten la capa externa del virus. Vacunas similares han sido desarrolladas previamente contra el virus del SARS, cuya eficacia ha sido variable en monos, pero no ha sido probada en humanos. De las cuatro proteínas estructurales presentes en SARS-CoV-2, la proteína S (spike) se encarga de la entrada del virus a la célula, y es quizás la candidata principal a ser utilizada como base, a partir de lo aprendido durante el desarrollo de la vacuna contra el SARS. Por su naturaleza, tal tipo de vacuna suele requerir dosis de refuerzo para alcanzar eficacia.

Adicional al uso de proteínas virales, podría optarse por el uso de vectores virales (con o sin capacidad de replicación), uso de ácidos nucleicos (altamente experimentales al momento), virus inactivados (recordando las bases de la vacuna contra la influenza estacional de

Tabla 2: Estudios clínicos con reclutamiento activo y registro en http://clinicaltrials.gov al 3 de junio de 2020.

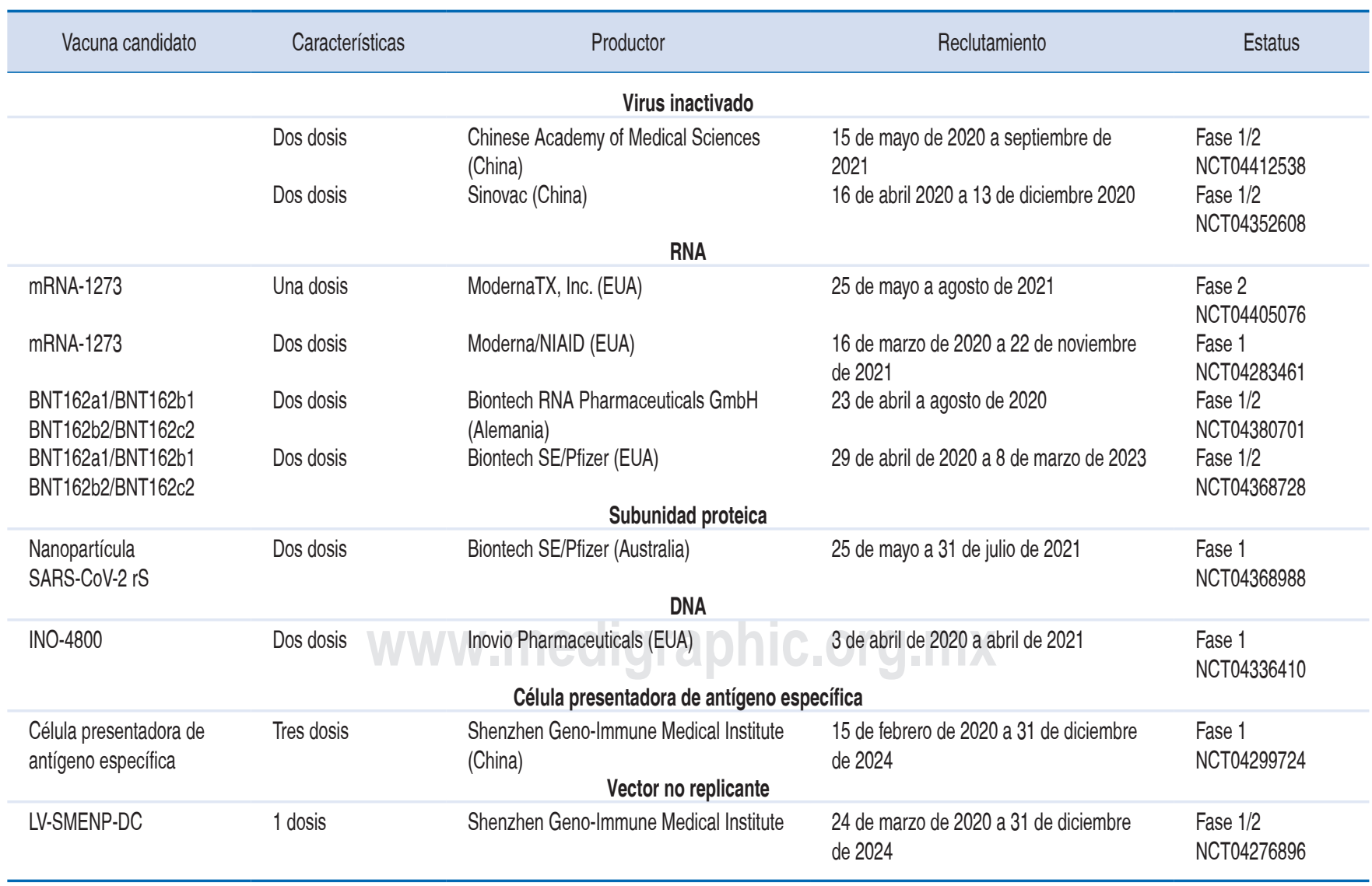


mayor uso a nivel mundial y la única recomendada para los receptores de trasplante) y virus vivos atenuados.

Con base en lo aprendido con la influenza, el desarrollo de vacunas de virus inactivados y atenuados es una de las opciones más factibles en el caso de SARS-CoV-2, teniendo como objetivo el desarrollo de patogénesis atenuada con daño pulmonar mínimo, limitado flujo de neutrófilos y una mayor proporción de citocinas antiinflamatorias. De esta manera, se buscaría disminuir el número de casos graves y la mortalidad asociada. ${ }^{36}$

Vacuna para SARS-CoV-2 y

dificultades a enfrentar

Dos de las mayores dificultades que se enfrentan durante el desarrollo de una nueva vacuna son la enorme inversión económica que representa para la industria y el factor tiempo. Conjugadas, la inversión suele ser cercana al billón de dólares sin garantía alguna de éxito en ningún momento (posibilidad de falla de hasta $94 \%) .{ }^{37}$

Previo a la llegada de SARS-CoV-2, gran parte de la información sobre la patogénesis de los coronavirus era proveniente de la medicina veterinaria. En tal campo, han existido múltiples intentos de vacunación, tanto vía entérica como parenteral. A pesar de esto, no se ha podido frenar del todo la pérdida de animales por coronavirus, lo cual tiene un impacto económico importante..$^{38}$ Por otro lado, la transmisión entre especies retrasa aún más la posibilidad de una vacuna universalmente eficaz.

Es reconocido que los coronavirus suelen causar inmunidad de corta duración y bajo nivel. ${ }^{39}$ Particularmente, en el caso de COVID-19 por medio de la medición directa de anticuerpos IgG e IgM a través de ELISA. En pacientes convalecientes tras la infección, se reportaron niveles detectables de IgG a partir del décimo día de la aparición de los síntomas; sin embargo, para la octava semana, el título de anticuerpos alcanzado empezó a descender. ${ }^{40}$ De manera relevante, en seis de 40 pacientes no se registraron anticuerpos detectables, aunque un punto relevante a considerar es la sensibilidad de las pruebas disponibles al momento y el nivel de respuesta inmunológica tras la exposición al virus.

Dentro de las dificultades halladas, deben considerarse la eficacia estimada de la vacunación y la transmisibilidad del virus. En el caso de la influenza, desde 2012 se recomienda que se vacune anualmente cuatrivalentemente; sin embargo, un metaanálisis de tal año estimó una eficacia de $59 \%$ en personas sanas de 19 a 65 años. ${ }^{41}$ Tales datos son escasos en la población receptora de trasplante de órgano sólido, en donde se ha registrado seroconversión en, al menos, un antígeno de la vacuna en el $55.8 \%$ de los casos. ${ }^{42} \mathrm{La}$ transmisibilidad suele expresarse por medio del ritmo o número reproductivo básico (R0), el cual representa el número de personas que pueden contagiarse a partir de un caso aislado; éste nos habla de la velocidad de propagación del virus. El grupo italiano, una de las poblaciones mayormente afectadas por SARS-CoV-2, ha estimado un R0 de 2.43 a $3.10^{43}$ comparado con el pico de influenza por H1N1 en 2009 (R0 estimado de 1.5). ${ }^{44}$ De esta manera, si lográramos la vacunación del $100 \%$ de la población, con una efectividad estimada de la vacuna de 50\%, SARS-CoV-2 aún mantendría un R0 superior a 1, comprometiendo el control adecuado de la expansión de la infección.

Si consideramos adicionalmente que la tasa de vacunación, descrita en los receptores de trasplante, puede ser tan baja como $52 \%$, estamos lejos de alcanzar el control absoluto de la infección a pesar de la disponibilidad de la vacuna. ${ }^{41}$

\section{La premura de desarrollar una vacuna y sus riesgos}

La morbilidad, mortalidad, impacto social y devastación financiera que ha traído consigo el SARS-CoV-2 ha trastornado la vida del mundo entero. En tal contexto, la presión sobre la comunidad científica para la pronta disposición de una vacuna efectiva, nunca había sido tan intensa. De esta manera, resulta tentador disponer de una vacuna en meses, a pesar de que sabemos que el tiempo usual de fabricación y validación de una nueva vacuna se suele medir en años. El método científico exige disciplina y rigor, lo cual lleva tiempo.

La premura por tener métodos preventivos eficaces de manera global puede poner en riesgo la calidad del producto final. Por el contrario, hay mucho que perder. Por un lado, la historia nos ha enseñado las consecuencias de la falta de cuidado de la calidad en los productos que se ponen a disposición de todo el mundo; en sus orígenes, la distribución de una marca contaminada de vacuna de virus vivo atenuado frente a la polio provocó debilidad muscular en 70 mil niños, 164 quedaron permanentemente paralizados y se registraron 10 fallecimientos. ${ }^{45}$ Adicional a los eventos adversos, como comunidad médica, tenemos sólo una oportunidad para ganarnos la confianza de la pobla- 
ción en la vacunación contra COVID-19. Si falláramos al ofrecer una vacunación poco eficaz o con efectos adversos serios (quizás consecuencia de la falta de regulación por la premura), comprometeríamos aún más la ya frágil confianza global en la vacunación. De manera indirecta, las tasas de vacunación en otras patologías, como la influenza, podría disminuir masivamente, con incrementos inaceptables en muertes prevenibles. ${ }^{46}$

De esta manera, hay mucho que perder al apresurarnos y poner a disposición masiva una vacuna que no sea meticulosamente evaluada y que pase por los protocolos de seguridad habituales, los cuales suelen durar años.

\section{Puntos positivos de una vacunación universal}

Aunque no se lograría controlar de manera absoluta la transmisibilidad del virus, podríamos incrementar la proporción de casos leves o asintomáticos que no requieran hospitalización y se reduciría la mortalidad, de la misma manera como se demostró con influenza. ${ }^{47}$

Contrario a lo registrado al momento en cuanto a la duración de los anticuerpos IgG protectores frente a SARS-CoV-2 (ocho semanas), se ha reportado que pacientes afectados durante el pico de SARS-CoV en la temporada 2002-2003 mantienen títulos de $\lg G$ a 12 años de seguimiento. ${ }^{48}$ Dada la alta similitud entre el virus, y a pesar de no contar con una vacuna universal y eficiente contra tal cepa, abre la posibilidad del desarrollo de una vacuna con efecto protector a largo plazo; adicionalmente, la menor variabilidad genética de esta cepa en relación con el comportamiento conocido de la influenza estacional abre la posibilidad de contar con una vacuna útil por varios años.

\section{CONCLUSIONES}

La llegada de SARS-CoV-2 ha trastornado el mundo por su impacto financiero, social, económico y por su gran morbilidad y mortalidad. Dada la ausencia de un tratamiento efectivo al momento, no deben subestimarse los esfuerzos enfocados a aplicar medidas preventivas de manera eficiente. El lavado de manos ha pasado la prueba del tiempo como la medida preventiva más costo-efectiva, tanto para SARS-CoV-2 como para muchas otras enfermedades. Adicionalmente, cada vez es más fuerte la evidencia que apoya el uso universal de mascarillas como medida poblacional.

Con base en nuestro aprendizaje previo, pareciera que sólo el disponer de vacunación universal cambiará la historia natural de la enfermedad. De esta manera, la comunidad científica se enfrenta actualmente al enorme reto de crear lo más rápido una vacuna eficiente, segura y accesible para el mundo. A pesar de la urgencia, no puede olvidarse la importancia del rigor científico para garantizar un producto de gran calidad que fortalezca la ya debilitada confianza global en la vacunación.

\section{REFERENCIAS}

1. Dexter HM. Using effective hand hygiene practice to prevent and control infection. Nursing Standard. 2020; 35 (5): 45-50.

2. Pires $D$, Pittet $D$. Hand hygiene mantra: teach, monitor, improve and celebrate. J Hosp Infect. 2017; 95 (4): 335-337.

3. Loveday HP, Wilson JA, Pratt RJ, Golsorkhi M, Tingle A, Bak A et al. Epic3: National evidence-based guidelines for preventing healthcare-associated infections in NHS hospitals in England. J Hosp Infect. 2014; 86: S1-S70.

4. Centers for Disease Control and Prevention. CDC Statement for Healthcare Personnel on Hand Hygiene during the Response to the International Emergence of COVID-19. 2020. Disponible en: $\quad$ www.cdc.gov/coronavirus/2019-ncov/hcp/hand-hygiene. html2020

5. Treas LS, Wilkinson JM, Barnett KL, Smith MH. Basic nursing: thinking, doing, and caring. 2a. ed. Philadelphia: F. A. Davis; 2010.

6. World Health Organization. WHO guidelines on hand hygiene in health care. 2009.

7. Huang $\mathrm{C}, \mathrm{Ma} \mathrm{W}$, Stack S. The hygienic efficacy of different hand-drying methods: a review of the evidence. Mayo Clin Proc. 2012; 87 (8): 791-798.

8. Murillo-Godínez G. The Flügge's drops. Rev Med Inst Mex Seguro Soc. 2009; 47 (3): 290.

9. Somsen GA, van Rijn C, Kooij S, Bem RA, Bonn D. Small droplet aerosols in poorly ventilated spaces and SARS-CoV-2 transmission. Lancet Respir Med. 2020.

10. Radonovich LJ, Simberkoff MS, Bessesen MT, Brown AC, Cummings DAT, Gaydos CA et al. N95 respirators vs medical masks for preventing influenza among health care personnel: a randomized clinical trial. JAMA. 2019; 322 (9): 824-833.

11. Eikenberry SE, Mancuso M, Iboi E, Phan T, Eikenberry K, Kuang $\mathrm{Y}$ et al. To mask or not to mask: Modeling the potential for face mask use by the general public to curtail the COVID-19 pandemic. Infect Dis Model. 2020; 5: 293-308.

12. World Health Organization. Advice on the use of masks in the context of COVID-19: interim guidance. 2020. Disponible en: https://apps.who.int/iris/handle/10665/331693

13. Lo JY, Tsang TH, Leung YH, Yeung EY, Wu T, Lim WW. Respiratory infections during SARS outbreak, Hong Kong, 2003. Emerg Infect Dis. 2005; 11 (11): 1738-1741.

14. Syed Q, Sopwith W, Regan M, Bellis MA. Behind the mask. Journey through an epidemic: some observations of contrasting public health responses to SARS. J Epidemiol Community Health. 2003; 57 (11): 855-856.

15. Jefferson T, Foxlee R, Del Mar C, Dooley L, Ferroni E, Hewak B et al. Physical interventions to interrupt or reduce the spread of respiratory viruses: systematic review. BMJ. 2008; 336 (7635): 77-80.

16. Burgess A, Horii M. Risk, ritual and health responsibilisation: Japan's 'safety blanket' of surgical face mask-wearing. Sociol Health IIIn. 2012; 34 (8): 1184-1198. 
17. Maclntyre CR, Cauchemez S, Dwyer DE, Seale H, Cheung P, Browne $G$ et al. Face mask use and control of respiratory virus transmission in households. Emerg Infect Dis. 2009; 15 (2): 233241.

18. Liang M, Gao L, Cheng C, Zhou Q, Uy JP, Heiner K et al. Efficacy of face mask in preventing respiratory virus transmission: A systematic review and meta-analysis. Travel Med Infect Dis. 2020; 101751.

19. Leung NHL, Chu DKW, Shiu EYC, Chan KH, McDevitt JJ, Hau BJP et al. Respiratory virus shedding in exhaled breath and efficacy of face masks. Nat Med. 2020; 26 (5): 676-680.

20. 3M. Decontamination methods for 3 M N95 respirators. Technical Bulletin; 2020 [citado el 4 de abril de 2020]. Disponible en: https:// multimedia.3m.com/mws/media/18248690/decontaminationmethods-for-3m-n95- respirators-technical-bulletin.pdf

21. Tran K, Cimon K, Severn M, Pessoa-Silva CL, Conly J. Aerosol generating procedures and risk of transmission of acute respiratory infections to healthcare workers: a systematic review. PLoS One. 2012; 7 (4): e35797.

22. Cook TM. Personal protective equipment during the coronavirus disease (COVID) 2019 pandemic - a narrative review. Anaesthesia. 2020; 75 (7): 920-927.

23. World Health Organization. Modes of transmission of virus causing COVID-19: implications for IPC precaution recommendations [Accesado el 31de marzo de 2020]. Disponible en: https://www.who.int/news-room/commentaries/ detail/modes-of-transmission-of-virus-causing-covid-19implications-for-ipc-precaution-recommendations

24. Ağalar C, Öztürk Engin D. Protective measures for COVID-19 for healthcare providers and laboratory personnel. Turk J Med Sci. 2020; 50 (SI-1): 578-584.

25. Centers for Diseases Control and Prevention. Interim Infection Prevention and Control Recommendations for Patients with Suspected or Confirmed Coronavirus Disease 2019 (COVID-19) in Healthcare Settings. Disponible en: https://www.cdc.gov/ coronavirus/2019-ncov/hcp/infection-control-recommendations. $\mathrm{html}$

26. BSI group. Respiratory protective devices. Filtering half masks to protect against particles. Requirements, testing, marking. 2020 [Accesado el 28 de marzo de 2020]. Available in: https:// www.bsigroup.com/en-GB/topics/novel-coronavirus-covid-19/ medical-devices-ppe/

27. NIOSH Guide to the Selection and Use of Particulate Respirators. 2020 [Access 26/3/2020]. Disponible en: https://www.cdc.gov/ niosh/docs/96-101/default.html

28. Seah I, Agrawal R. Can the coronavirus disease 2019 (COVID-19) affect the eyes? a review of coronaviruses and ocular implications in humans and animals. Ocul Immunol Inflamm. 2020; 28 (3): 391-395.

29. van Doremalen N, Bushmaker T, Morris DH et al. Aerosol and surface stability of SARS-CoV-2 as compared with SARSCoV-1. N Engl J Med. 2020; 382 (16): 1564-1567.

30. Cohen J. Vaccine designers take first shots at COVID-19. Science. 2020; 368 (6486): 14-16.

31. Billington J, Deschamps I, Erck SC et al. Developing vaccines for SARS-CoV-2 and future epidemics and pandemics: applying lessons from past outbreaks. Health Secur. 2020.

32. Hirzel C, Kumar D. Influenza vaccine strategies for solid organ transplant recipients. Curr Opin Infect Dis. 2018; 31 (4): 309315.

33. Kumar D, Campbell P, Hoschler K, Hidalgo L, Al-Dabbagh M, Wilson $L$ et al. Randomized controlled trial of adjuvanted versus nonadjuvanted influenza vaccine in kidney transplant recipients. Transplantation. 2016; 100 (3): 662-669.

34. Cordero E, Roca-Oporto C, Bulnes-Ramos A et al. Two doses of inactivated influenza vaccine improve immune response in solid organ transplant recipients: results of TRANSGRIPE 1-2, a randomized controlled clinical trial. Clin Infect Dis. 2017; 64 (7): 829-838.

35. Callaway E. The race for coronavirus vaccines: a graphical guide. Nature. 2020; 580 (7805): 576-577.

36. Shang W, Yang Y, Rao Y, Rao X. The outbreak of SARS-CoV-2 pneumonia calls for viral vaccines. NPJ Vaccines. 2020; 5: 18.

37. Pronker ES, Weenen TC, Commandeur H, Claassen EH, Osterhaus AD. Risk in vaccine research and development quantified. PLoS One. 2013; 8 (3): e57755.

38. Decaro N, Martella V, Saif LJ, Buonavoglia C. COVID-19 from veterinary medicine and one health perspectives: What animal coronaviruses have taught us. Res Vet Sci. 2020; 131: 21-23.

39. Fung TS, Liu DX. Human Coronavirus: Host-Pathogen Interaction. Annu Rev Microbiol. 2019; 73: 529-557.

40. Adams ER, Anand R, Andersson MI, Auckland K, Baillie JK, Barnes $E$ et al. Evaluation of antibody testing for SARS-Cov-2 using ELISA and lateral flow immunoassays. medRxiv. 2020.

41. Harris K, Baggs J, Davis RL, Black S, Jackson LA, Mullooly JP et al. Influenza vaccination coverage among adult solid organ transplant recipients at three health maintenance organizations, 1995-2005. Vaccine. 2009; 27 (17): 2335-2341.

42. Natori Y, Shiotsuka M, Slomovic J et al. A Double-blind, randomized trial of high-dose vs standard-dose influenza vaccine in adult solid-organ transplant recipients. Clin Infect Dis. 2018; 66 (11): 1698-1704.

43. D'Arienzo M, Coniglio A. Assessment of the SARS-CoV-2 basic reproduction number, $\mathrm{R} 0$, based on the early phase of COVID-19 outbreak in Italy. Biosaf Health. 2020.

44. Ridenhour B, Kowalik JM, Shay DK. Unraveling $R_{0}$ : considerations for public health applications. Rev Panam Salud Publica. 2015; 38 (2): 167-176.

45. Treanor J. The cutter incident: how America's first polio vaccine led to the growing vaccine crisis. Environ Health Perspect. 2006; 114 (9): A556.

46. Trogen B, Oshinsky D, Caplan A. Adverse consequences of rushing a SARS-CoV-2 vaccine: implications for public trust. JAMA. 2020.

47. Thompson MG, Pierse N, Sue Huang $Q$ et al. Influenza vaccine effectiveness in preventing influenza-associated intensive care admissions and attenuating severe disease among adults in New Zealand 2012-2015. Vaccine. 2018; 36 (39): 5916-5925.

48. Guo X, Guo Z, Duan C, Chen Z, Wang G, Lu Y et al. Long-term persistence of igg antibodies in SARS-CoV infected healthcare workers. medRxiv. 2020

\section{Correspondencia:}

Dra. Mónica Sánchez-Cárdenas

Área de Nefrología del Trasplante.

Departamento de Nefrología y Metabolismo

Mineral.

Instituto Nacional de Ciencias Médicas y Nutrición

«Salvador Zubirán».

Vasco de Quiroga Núm. 15,

Belisario Domínguez, Sección XVI,

Tlalpan, 14080, Ciudad de México.

Teléfono: +52 (55) 5513-5827

E-mail:dra_monicasanchez@hotmail.com 